\title{
COHERENT SCATTERERS DETECTION: APPLICATION OVER GLACIER TERRAIN USING TERRASAR-X TIME SERIES DATA
}

\author{
Maria J. Sanjuan-Ferrer ${ }^{1}$, Irena Hajnsek ${ }^{1,2}$, Kostantinos P. Papathanassiou ${ }^{1}$ \\ ${ }^{1}$ German Aerospace Center - Microwaves and Radar Institute \\ PO BOX 1116, 82230, Wessling, Germany \\ ${ }^{2}$ ETH Zurich - Institute of Environmental Engineering \\ Wolfgang-Pauli-Str. 15, CH-8093 Zurich \\ \{maria.sanjuanferrer, irena.hajnsek, kostas.papathanassiou\}@dlr.de
}

\begin{abstract}
This paper investigates the detection of Coherent Scatterers (CSs) in ice and glacier terrain by means of TerraSAR-X time series data in the test site of the Helheim Glacier in Greenland. CSs are evaluated with respect to detection and potential applications. Applying optimized detection scheme can be useful for retrieving information about the glacier movement using time series data. Finally, some conclusions about the temporal stability of natural CSs are obtained by analyzing the rocky area around the glacier.
\end{abstract}

Index Terms - Coherent Scatterers, Synthetic Aperture Radar (SAR), glacier terrain, TerraSAR-X, time series data.

\section{INTRODUCTION}

The interest in using Synthetic Aperture Radar (SAR) to study and monitor ice and glacier terrain for glaciological and climate change research has increased in the last years. These distributed areas are characterized by complex scattering mechanisms and, at the same time, they are affected by different temporal and environmental effects (i.e. snowfall, melting...) which makes it necessary to take them into account.

Recently, the Coherent Scatterers (CSs) technique was introduced in order to detect scatterers with a deterministic point-like scattering behaviour (commonly associated with high scattering amplitudes, deterministic phases, high spectral correlation and high interferometric coherence values) in different natural and urban scattering scenarios, using a single SAR image [1]. These kinds of scatterers are valuable for a wide spectrum of applications, ranging from image calibration to object characterization and information extraction. In the special case of a natural volume scatterers like ice, the CSs detection can be possible in textured/featured regions like on borders of crevasses and moraine materials on glaciers.

In this work, the detection of CSs is investigated by means of wideband spaceborne SAR systems in ice and glacier terrain, and the properties and potential applications of the detected CSs are analysed when time series data of the same scene are available.

\section{CSS DETECTION}

CSs are characterised by a stable spectral correlation. Accordingly, spectral correlation techniques have been proposed for their detection. Three different approaches can be found in the literature:

1. The originally proposed sublook coherence detection based on the cross-correlation of two spectral sub-bands [1]. Scatterers with high sublook correlation values are considered as detected CSs;

2. the sublook entropy detection based on the crosscorrelation of multiple spectral sub-bands [1]. In this case, scatterers with low sublook entropy values are considered as detected CSs. This method allows a more flexible detection with respect to the spectral characteristics of the individual CSs;

3. the phase variance approach [3]-[4]. This technique uses as a detector the stability of the phase along range/azimuth frequency and allows a widely preservation of the spatial resolution in the detection of CSs.

Each approach has its own pros and cons. However, common to all of the three approaches is the trade-off between spatial resolution (defined by the bandwidth of the individual sub-bands and the number of samples used) and the quality of the detected CSs. 

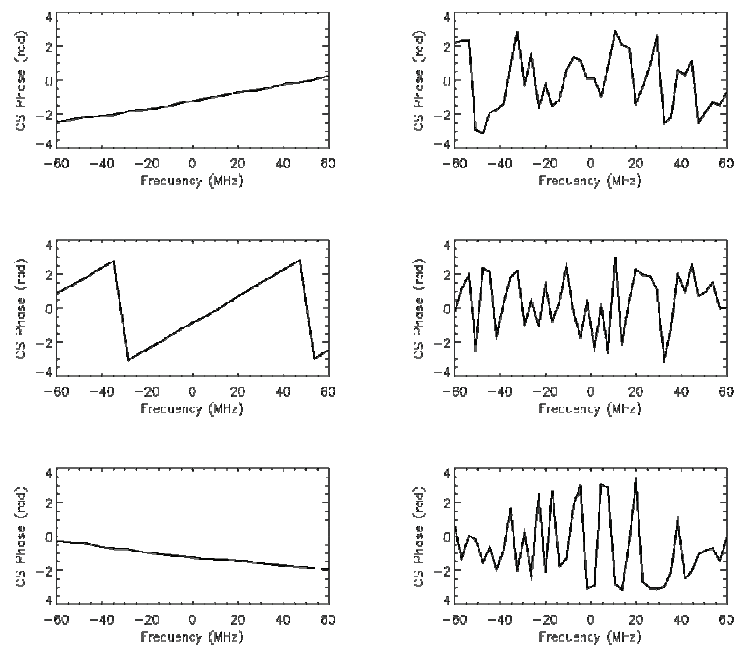

(a)

(b)

Figure 1 - Examples of the phase as a function of frequency for: (a) three CSs; (b) three non-CSs.

\section{REAL DATA RESULTS}

The considered test site is the Helheim glacier, located in the south-east of Greenland $\left(66^{\circ} 21^{\prime} \mathrm{N} 38^{\circ} 12^{\prime} \mathrm{W}\right)$. It is the largest outlet glacier in Greenland and one of the fastest glaciers of the world moving several kilometres per year. After years of melting back at a relatively stable speed, Helheim has dramatically accelerated its retreat. The overall Greenland ice sheet contains approximately 10 percent of the world's freshwater and is up to two miles thick.

Our analysis is based on TerraSAR-X acquisitions performed between August 2008 and October 2009. The images were acquired every 11 days in the strip map mode, $\mathrm{HH}$ polarization, with a $150 \mathrm{MHz}$ range bandwidth and incidence angle of $37.3^{\circ}$.

The method considered for the CSs detection in the present work is the phase variance approach [2]-[3]. This detection method exploits the phase stability in the range/azimuth frequency. In fact, while the phase of the non-CSs varies randomly, even when using overlapping sublooks, the phase of the CSs changes almost linearly with the frequency. Such CSs linear behavior can be observed in Figure 1, in which three realizations observed in real data are reported of $\mathrm{CS}$ and non-CS phase history versus frequency.

This method can be applied in both range and azimuth directions. It has been shown in [4] that a combined rangeazimuth detection increases the signal-to-clutter ratio of the detected CSs and decreases the false alarm rates. In a first step, working along range, 10 sublooks of $60 \mathrm{MHz}$ each out of the available $150 \mathrm{MHz}$ range bandwidth have been generated, spanning a frequency range of $90 \mathrm{MHz}$. The phase derivative variance $\sigma^{2}$ rg has been computed for every

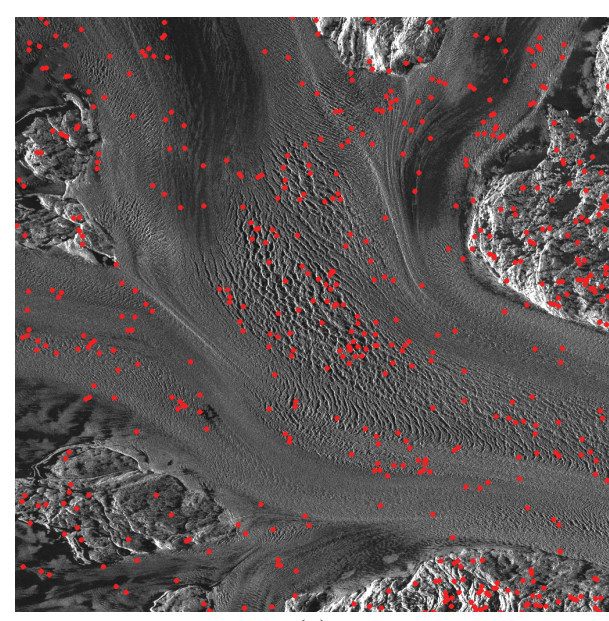

(a)

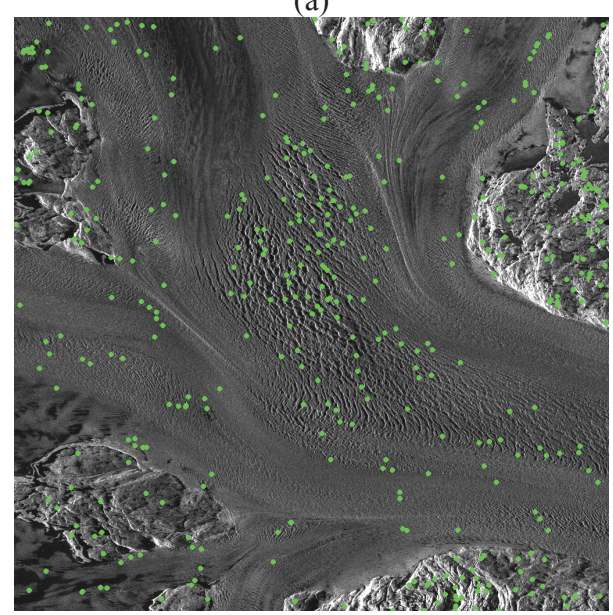

(b)

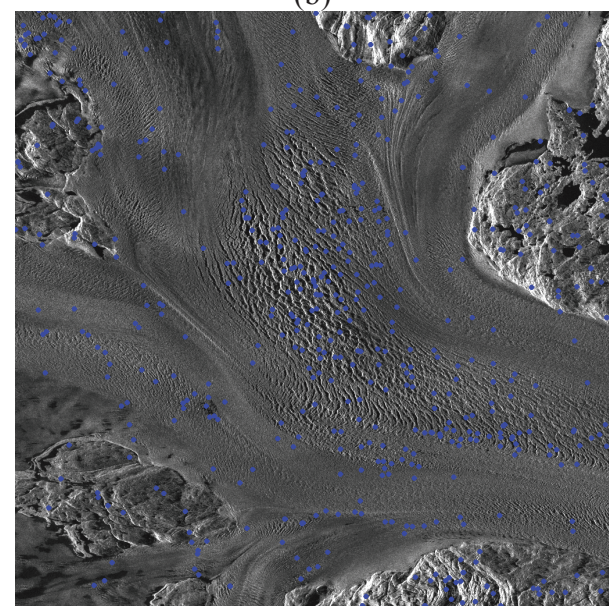

(c)

Figure 2 - Common range-azimuth CSs detected using the phase variance approach (threshold $\sigma^{2}<0.0025 \mathrm{rad}^{2}$ ). The range direction is on the horizontal axis. (a) In red, CSs detected in image 1 (16-06-2009): 450 points. (b) In green, CSs detected in image 2 (30-06-2009): 363 points. (c) In blue, CSs detected in image 3 (11-07-2009): 507 points. 


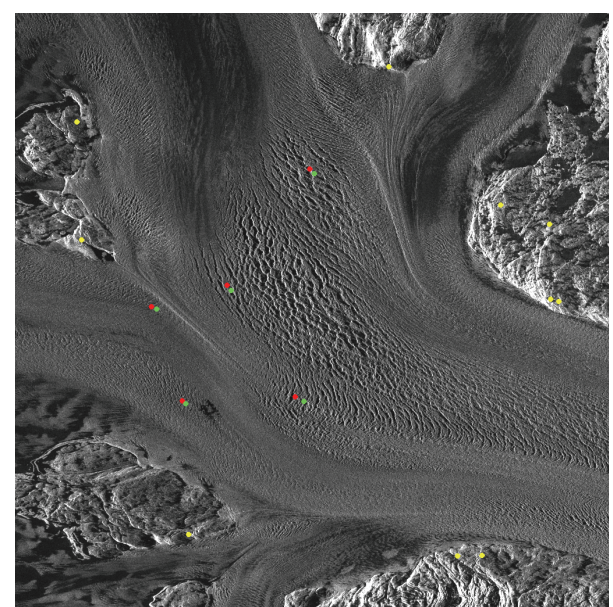

(a)

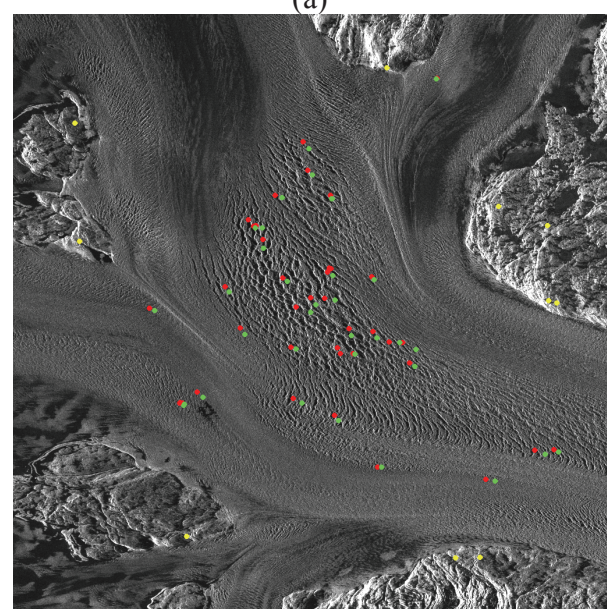

(b)

Figure 3 - Candidate pairs to be the same CS between two consecutive images (image 1 and image 2). (a) Candidate pairs between image 1 (red points) and image 2 (green points) with a window detection of the $10 \%$ of the shift in the area. (b) Candidates between image 1 and image 2 with a detection window of the $90 \%$.

pixel across these 10 sublooks. In a second step, the detection has been performed in azimuth: 10 sublooks of $1106 \mathrm{~Hz}$ each out of the $2765 \mathrm{~Hz}$ azimuth available bandwidth have been formed and the variance $\sigma_{\text {az }}^{2}$ of the phase derivative for every pixel across these 10 looks is estimated. In this way, fixing a threshold $\left(\sigma^{2}<0.0025 \mathrm{rad}^{2}\right)$, the pixels with $\sigma_{\mathrm{rg}}^{2}$ and $\sigma_{\mathrm{az}}^{2}$ at the same time lower than the fixed threshold will be considered as detected CSs. In Figure 2 the results of the common range-azimuth detection in three different acquisitions of the dataset (19 and 30 June 2009 and 11 July 2009) are shown.

As well, the possibility of retrieving information about the glacier movement has been investigated through an image pair by means of the detected CSs. Preliminary low resolution information regarding the shift in range and

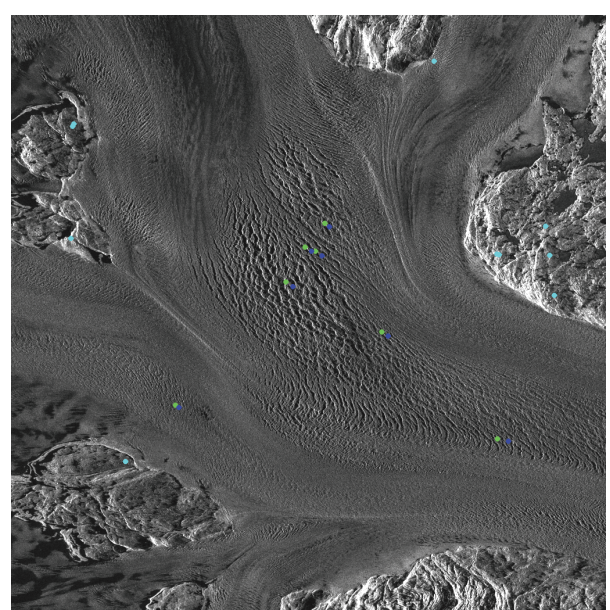

(a)

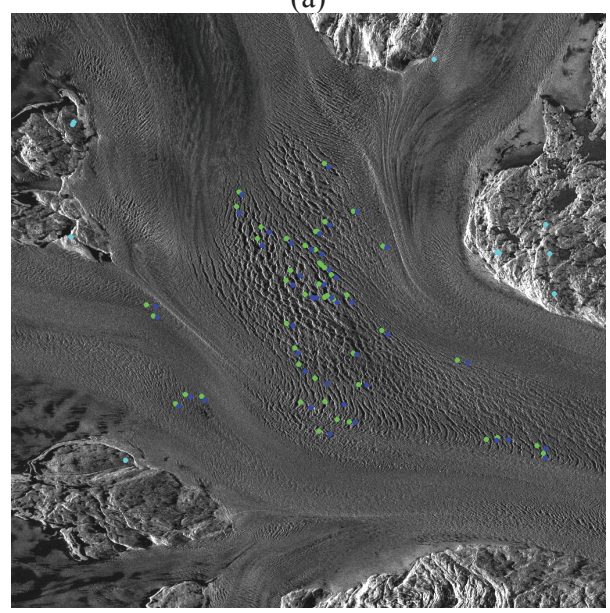

(b)

Figure 4 - Candidate pairs to be the same CS between two consecutive images (image 2 and image 3). (a) Candidate pairs between image 2 (red points) and image 3 (green points) with a window detection of the $10 \%$ of the shift in the area. (b) Candidates between image 2 and image 3 with a detection window of the $90 \%$.

azimuth between two images is employed (obtained from their cross-correlation) [5]. Given the range-azimuth shift map, for each detected CS in the first image it is possible to fix a range-azimuth window in which that CS is likely to be in the second image. Figure 3(a) shows the candidate pairs to be the same CS after 11 days, obtained with a window as big as the $10 \%$ of the shift. By increasing the detection window [e.g. 90\% in Figure 3(b)], the number of candidate pairs increases, at the cost of a possible increase of the number of the false pairs. Anyway, an indication has been obtained that a number of CSs can be retrieved in an image pair following the glacier movement. Similar results can be observed between the pair image 2 - image 3 in Figure 4.

In order to get more information about the stability of CSs in a glacier environment, the CSs detection was 


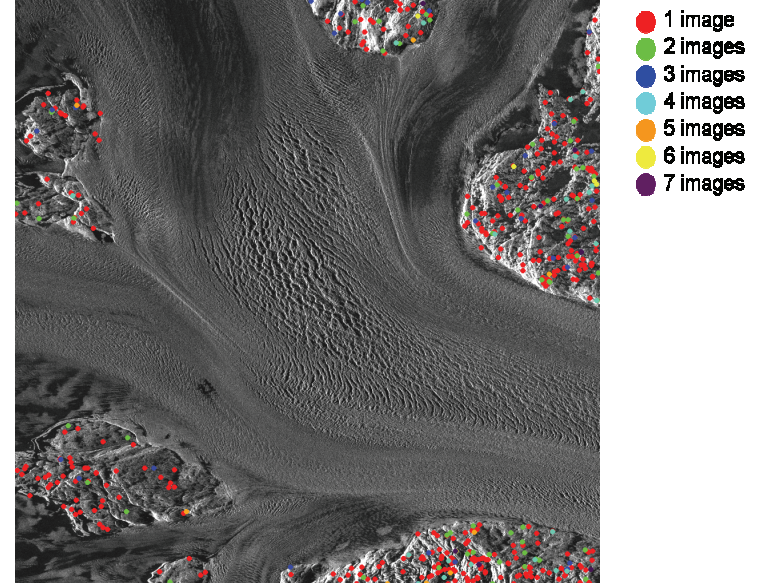

Figure 5 - Stable points in the rocks in long term period: from red points (detected CSs visible in only one image) to violet points (detected CSs visible in all seven images).

performed in a favorable region (with respect to the coherence change in time) of our test site: the rocky area. For such purpose, the temporal behavior in terms of detection for the CSs located on the rocks was studied, using 7 consecutive images (from 19 June 2009 to 24 August 2009, every 11 days). In Figure 5, the results obtained are plotted with different colors: from red (CSs are visible only in one image, or points that "disappear" in less than 11 days) to violet (CSs are visible in all seven images, or points that remain stable - at least - during 66 days).

Analogously, in Table 1 (a) are shown the number of detected CSs (using the same common range-azimuth phase variance detection, with threshold $\sigma^{2}=0.0025 \mathrm{rad}^{2}$ ) only on the rocky area for all the seven images. The decreasing tendency on the number of detected points probably is due to an increase of the temperatures, and consequently, the presence of water that makes more instable the scattering mechanism. Instead, in Table 1 (b) are shown the number of stable points in time. The majority of the points are red, which means that our detected CSs are only visible during less than 11 days. Remarkable is the fact that some of the cyan and orange points correspond not to consecutive stable points but detected points that disappeared for a period (11 or 22 days) and appeared later again.

\section{CONCLUSIONS}

The detection of CSs in glacier environments has been evaluated. The approach considered is the phase variance method [2]-[3], which exploits the high phase stability characteristic of the CSs along the frequency and independently of the direction (range or azimuth).

\begin{tabular}{lllllll}
\hline IM1 & IM2 & IM3 & IM4 & IM5 & IM6 & IM7 \\
\hline 227 & 175 & 137 & 123 & 130 & 137 & 135 \\
\hline
\end{tabular}

(a)

\begin{tabular}{llllllll}
\hline 0 & 0 & 0 & 0 \\
\hline 480 & 84 & 45 & 27 & 11 & 8 & 10 \\
\hline
\end{tabular}

(b)

Table 1 - (a) Number of detected CSs on the rocks area for seven consecutive images (from 19 June 2009 to 24 August 2009, every 11 days). (b) Number of stable CSs on the rocks area, from only one image (red) to all seven images (violet).

The potential of the common range-azimuth CSs detection in natural scenes [4] has been applied to investigate the possibility of tracking the displacement of some CSs with the movement of the glacier when the temporal separation between images is small (11 days).

In addition, experiments have shown that natural CSs have a limited "life time" due to the temporal and/or dynamic environmental effects within the scene. Such situation has been investigated looking to the stability of the CSs in the rocky area for a longer temporal period.

\section{REFERENCES}

[1] R. Zandona-Schneider, K. P. Papathanassiou, I. Hajnsek and A. Moreira: Polarimetric and Interferometric Characterization of Coherent Scatterers in Urban Areas. IEEE Trans. Geoscience and Remote Sensing, vol. 44, No. 4, April 2006.

[2] V.M. Giacovazzo, A. Refice, F. Bovenga and N. Veneziani: Identification of Coherent Scatterers: Spectral Correlation vs. Multi-Chromatic Phase Analysis. IGARSS'08, Boston, MA, USA, July 2008.

[3] R. Zandona-Schneider and K. P. Papathanassiou: Estimation and Correction of Ionospheric Induced Phase Errors in SAR images using Coherent Scatterers. IGARSS'09, Cape Town, South Africa, July 2009.

[4] M.J. Sanjuan-Ferrer, I. Hajnsek and K.P. Papathanassiou: Coherent Scatterers Detection in Glacier Environments by means of TerraSAR-X Images. EUSAR'2010, Aachen, Germany, June 2010.

[5] M. Villano et al.: SIGNAL: Mission Concept and Performance Assessment. EUSAR'2010, Aachen, Germany, June 2010. 